\title{
UNREDUCED ANTERIOR DISLOCATION OF THE HIP
}

\author{
Gawad Hamada, Alexandria, Egypt
}

Anterior dislocation of the hip is uncommon compared with posterior dislocation. Thompson and Epstein (1951) collected 204 cases of dislocation of the hip from the Los Angeles Hospital records in twenty-one years. Of these, 180 cases were posterior whereas only eighteen were anterior.

In the last ten years seven cases of anterior dislocation presented themselves to us. Three dislocations were acute and were reduced on admission. The remaining four were old unreduced dislocations.

Dislocations seen early are easily reduced by the well known manipulative methods. Old unreduced dislocations present much greater problems. A number of cases of unreduced posterior dislocation have been reported, but unreduced anterior dislocation is almost unheard of. Searching extensively in the literature the author has not come across any recorded case like those to be reported in this paper.

\section{MECHANISM OF INJURY}

Traumatic dislocation-Two forces may be responsible for the production of anterior dislocation. One is a force from the knee through the femur while the limb is in a position of extreme flexion, abduction and lateral rotation. This type of injury is exemplified by one of our patients who fell while riding astride on a donkey, her knee striking the ground (Case 1). The second type of injury is a force applied to the inner side of the thigh, forcing it into extreme lateral rotation and abduction, the limb being in a position of flexion. This type of injury was graphically related by one of our patients who was admitted with an acute anterior dislocation. He gave a history that he was supporting the weight of his body on his right flexed limb while the left limb was resting on a higher level in the position of flexion. He was piling sandbags, when suddenly the mound slipped and hit the inner part of his flexed thigh, felling him to the ground and resulting in an anterior dislocation.

Pathological dislocation-This may be caused by infantile paralysis, tumours or inflammatory conditions. The dislocation is produced through the prolonged maintenance of a position that facilitates slipping of the head of the femur anteriorly. This is shown in Case 2.

\section{CLINICAL FEATURES}

The patient presents an exaggerated lumbar lordosis with scoliosis towards the affected side compensating the hip deformity. The pelvis is lower on the affected side. The Trendelenburg test is positive in the old chronic cases. The limb is abducted, laterally rotated and slightly flexed at the hip and the knee.

In the pubic type of dislocation the head may be felt lying over the pubic bone under cover of the origin of the adductors. In the obturator type the head is not felt. The greater trochanter is difficult to palpate because it is pushed nearer to the midline and because the limb is markedly abducted.

Movements of the hip are generally lost, with the exception of flexion and abduction which are greatly reduced. Real lengthening is slight, but apparent lengthening is marked. The gait is laboured and the strides are short and jerky, the patient putting little weight on the affected side. In walking, the patient fixes the extended thigh by placing the hand over the knee, in the manner of a patient with quadriceps paralysis. Since the femoral head is displaced outside the acetabular socket, the abductor muscles become tense and cause flexion 


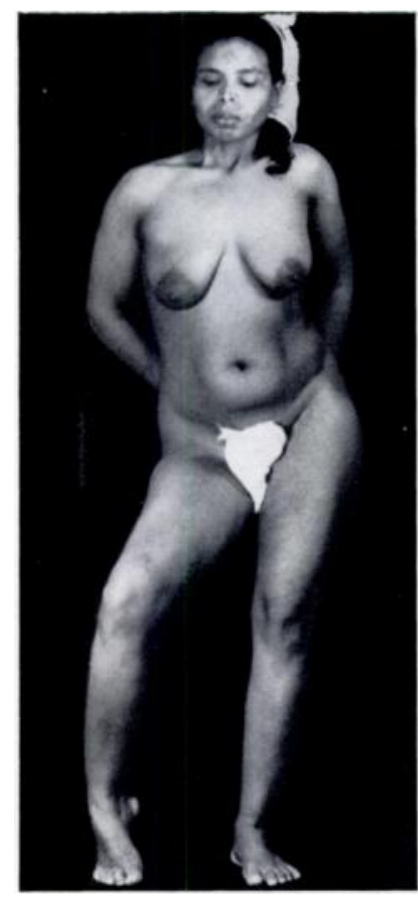

Fig. 1
Fig. 2

Fig. 2

Fig. 2

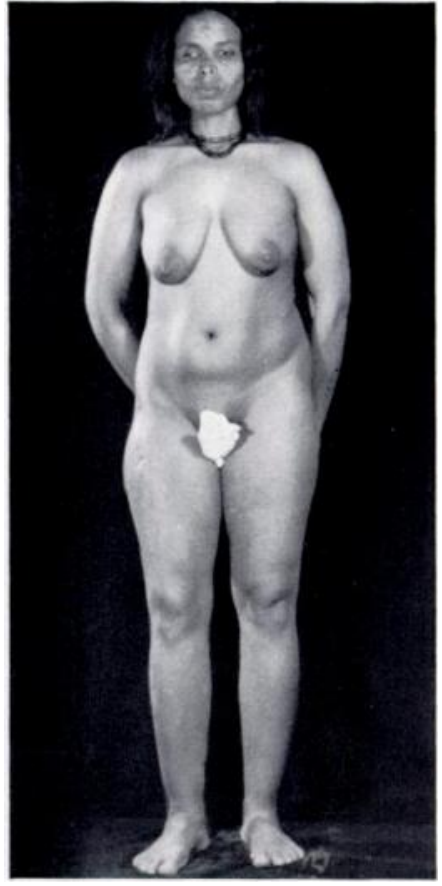

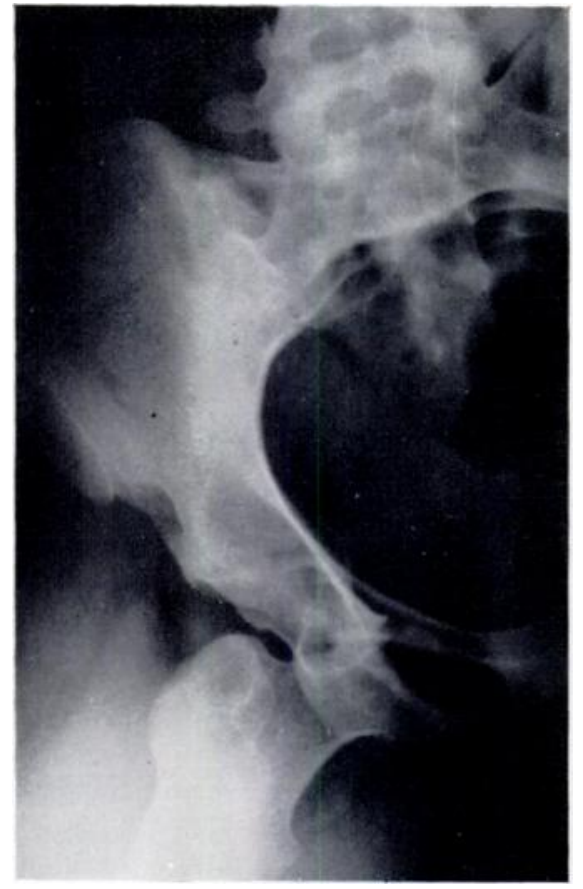

FIG. 3

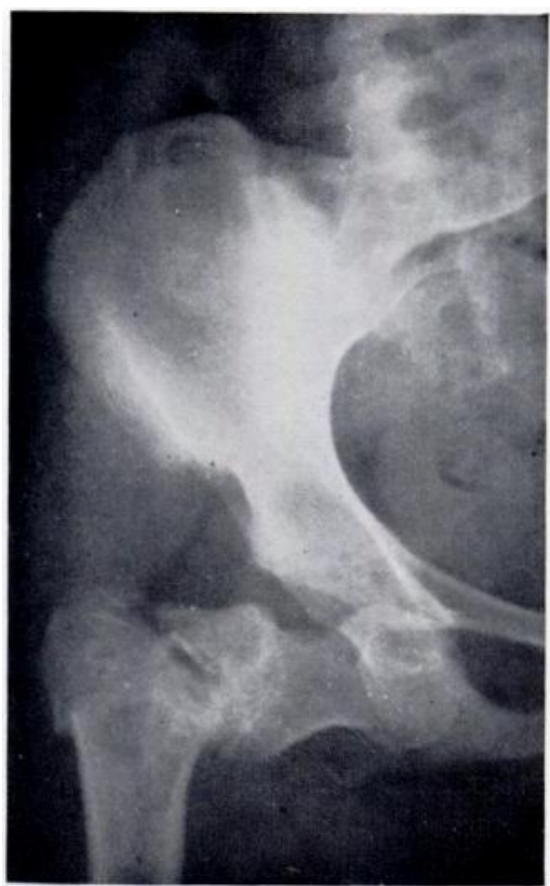

Fig. 4

Case 1-Radiographs before and after corrective osteotomy. 
of the hip. The pelvis rolls to the front; thus the Trendelenburg lurch in these cases occurs more towards the front than to the side. This explains why the patient places his hand on his thigh, to prevent further rolling forward of the pelvis, and thus attempts to stabilise his gait.

\section{TREATMENT}

The aim of any operative intervention is to correct the deformity and improve body mechanics and balance. An osteotomy is done at the base of the neck, from above downwards and medially. The limb is then slightly adducted, extended and medially rotated. The abductor muscles attached to the greater trochanter sometimes pull the shaft slightly upwards on the base of the neck, rendering the upper end of the femur L-shaped. This will give better stability to the pelvis, thus distributing the weight of the trunk on the affected side between the trochanter and the head, and not solely on the head (see Case 3). After operation the limb is placed in a plaster spica which includes the sound limb to the knee. Union is usually firm in three to four months.

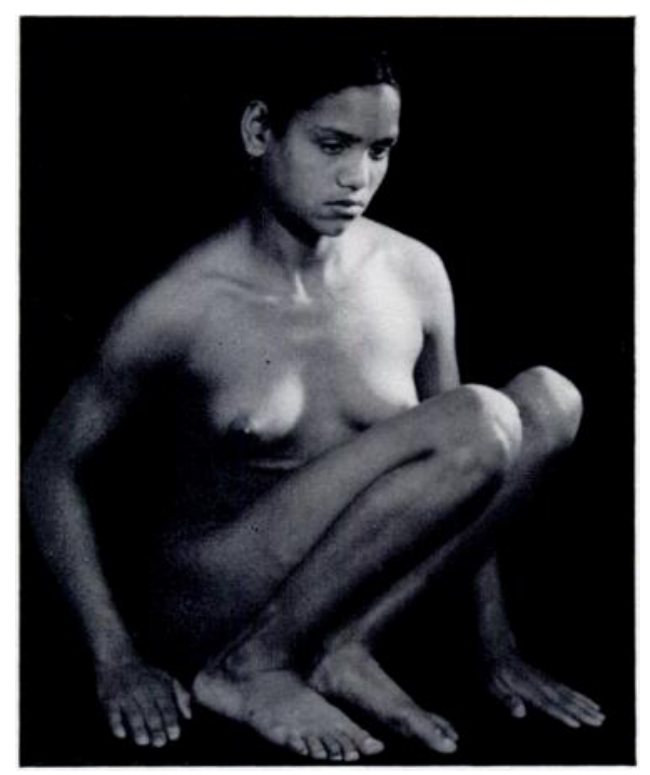

FIG. 5

Case 2-Attitude maintained by the patient during the active stage of the arthritis: abduction of the affected hip and adduction of the sound hip.

\section{CASE REPORTS}

Case 1-Woman aged twenty-four years. This patient had fallen from a donkey twelve years before. Radiographs (Fig. 3) showed the femoral head, small and atrophied, lying opposite the obturator foramen. The acetabulum and the iliac bone were undeveloped, probably because the injury had occurred at an early age, before union of the epiphysis. The femoral head was probably affected by avascular necrosis in consequence of the dislocation. Operation was done and resulted in the improvement of the deformity, stability and gait (Figs. 1 to 4 ).

Case 2-Girl aged sixteen years. The history was of pain in the left hip with rise of temperature. An abscess was incised. The patient remained in the sitting position for two years; the affected hip was abducted, laterally rotated, and touching the bed. The right hip was adducted and lay over the left thigh. Two years after the onset, when the inflammation had subsided, she gradually started to extend her limbs and when she stood up she found that she had developed the deformity. Osteotomy was done with improvement of the condition (Figs. 5 to 9).

vol. 39 B, No. 3, AUGUST 1957 


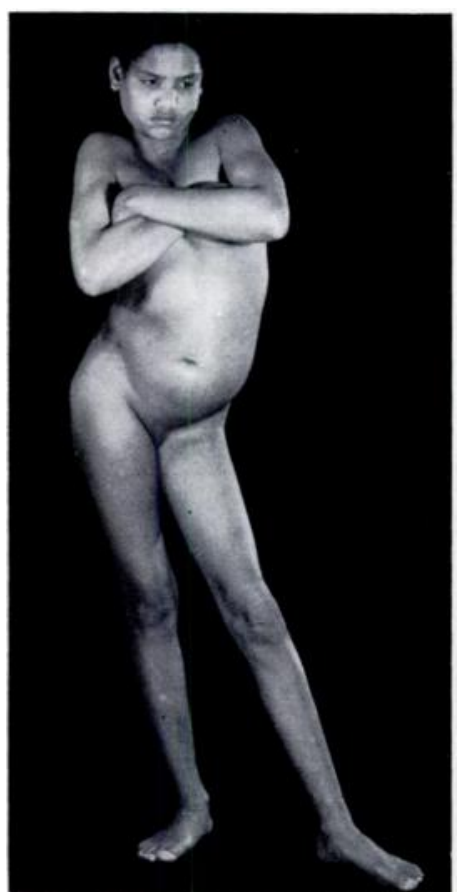

FIG. 6

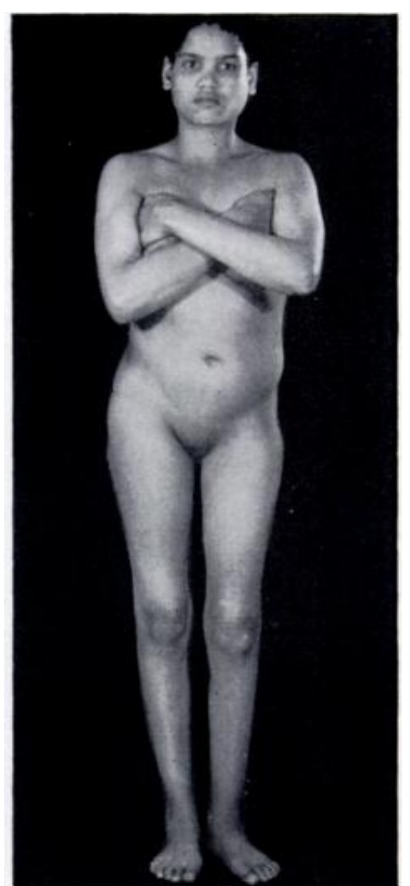

FIG. 7

Case 2-Photographs of the patient before and after corrective osteotomy.

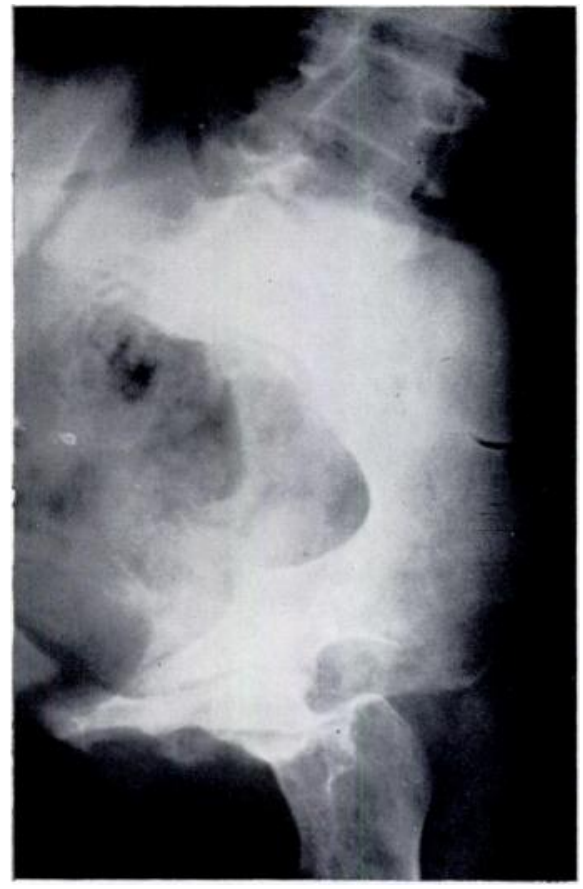

FIG. 8

Case 2-Radiographs before and after corrective osteotomy.

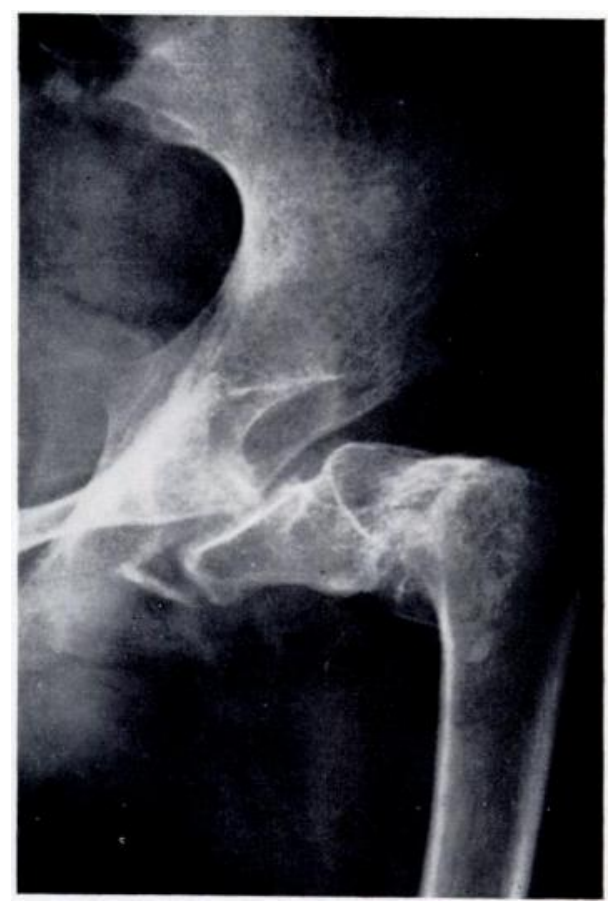

Fig. 9 


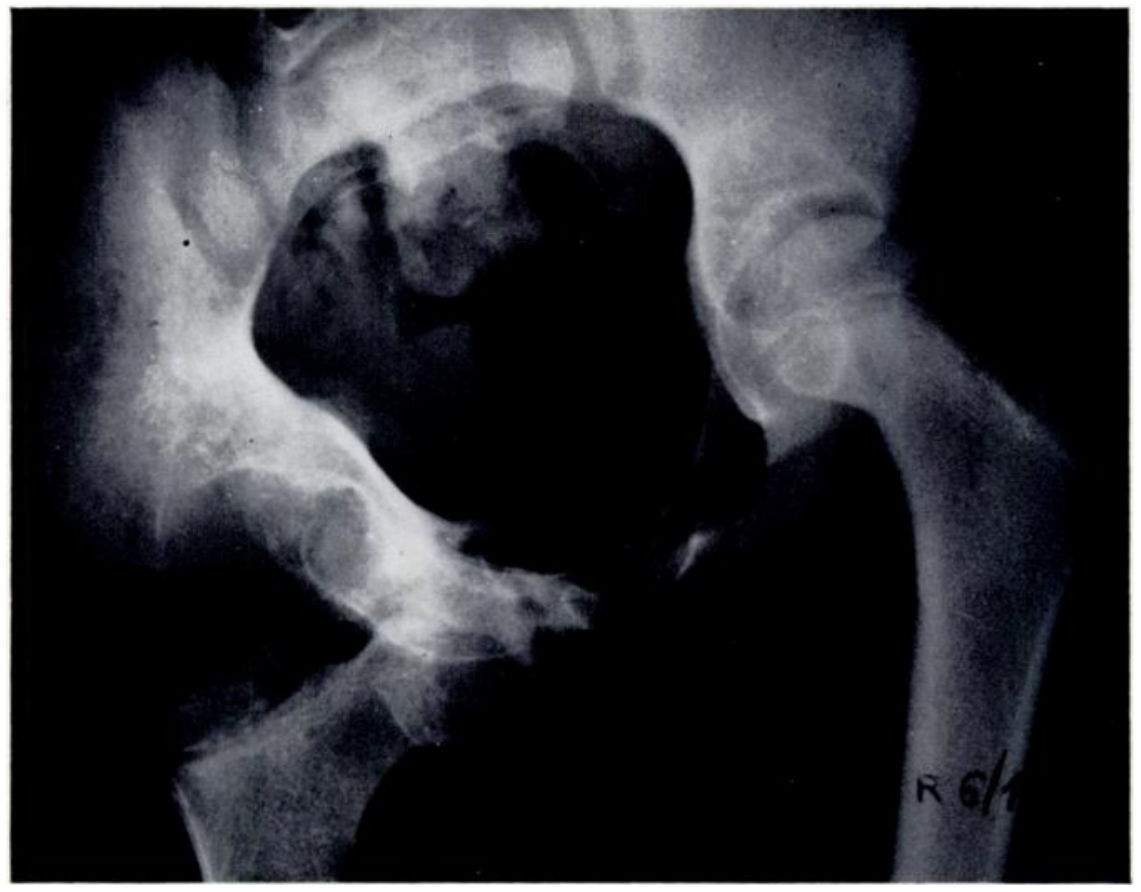

FIG. 10

Case 3-Radiograph before operation.

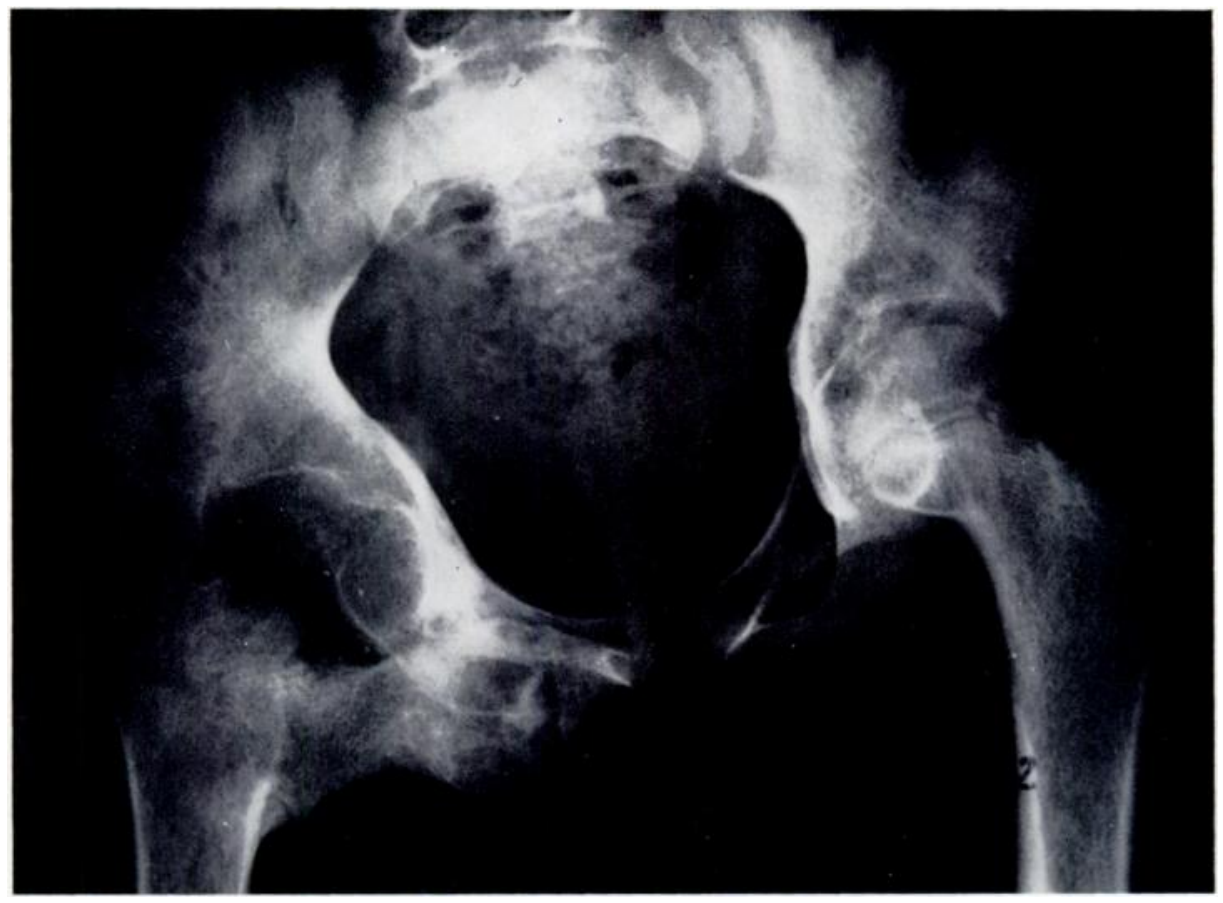

Fig. 11

Case 3-After corrective osteotomy.

VOL. 39 B, NO. 3, AUGUST 1957 
Case 3-Boy aged twelve years, who had fallen from a height a year before his admission to hospital. The deformity was the same as in the previous cases. Osteotomy was done and the deformity corrected (Figs. 10 and 11).

Case 4-Girl aged nine years. She had slipped and rolled down a flight of stairs five months before admission. Corrective osteotomy was done with satisfactory results (Fig. 12).

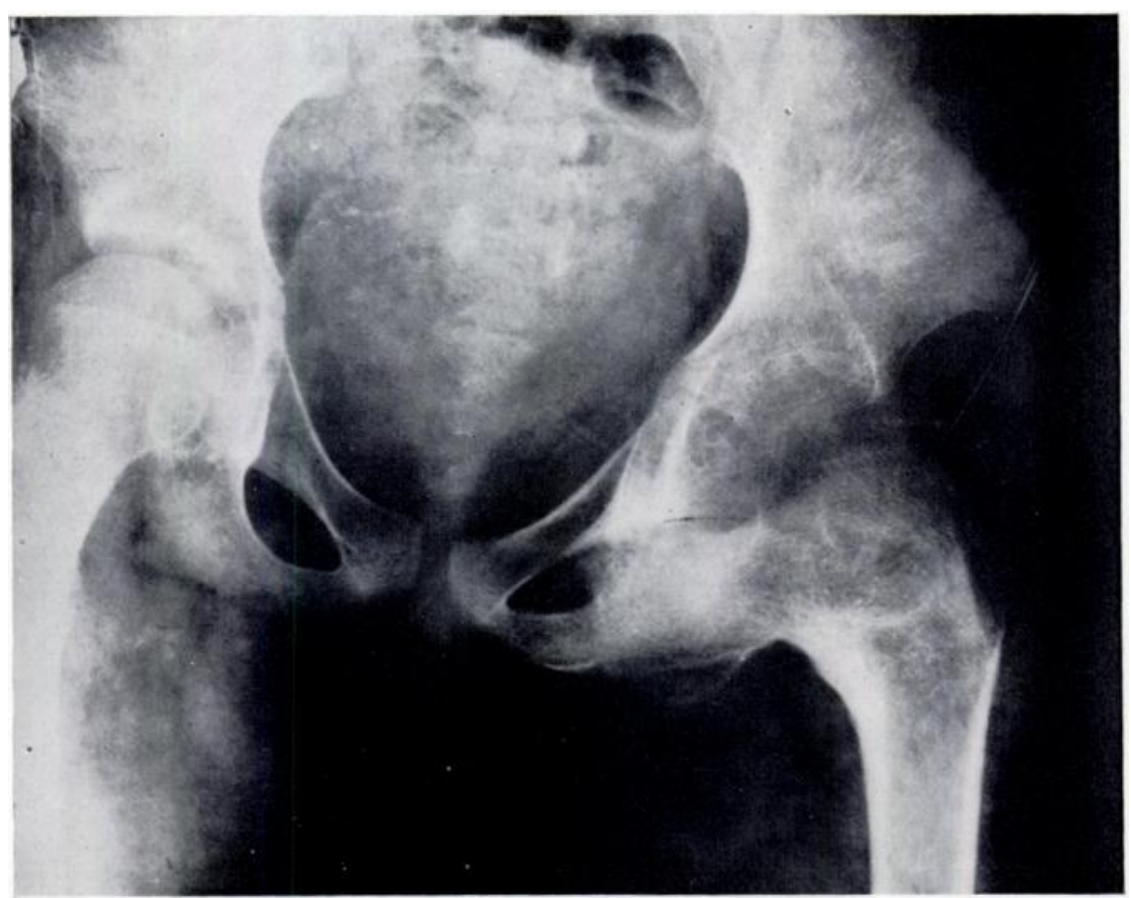

Fig. 12

Case 4-Radiograph after corrective osteotomy.

\section{SUMMARY}

1. Unreduced anterior dislocation of the hip is very uncommon. Four cases are reported. 2. The aim of treatment is to correct the deformity, to improve the gait and to produce pelvic stability.

3. Osteotomy of the femur at the trochanteric level is the simplest corrective operation and will greatly improve the function of the limb.

\section{REFERENCE}

Thompson, V. P., and Epstein, H. C. (1951): Traumatic Dislocation of the Hip. Journal of Bone and Joint Surgery, 33-A, 746. 